\title{
Experimental research on high pressure phase transitions of Mo and Ta*
}

\author{
L.-C. Cai ${ }^{\text {a }}$, Z.-Y. Zeng, X.-L. Zhang, and J.-B. Hu
}

National Key Laboratory for Shock Wave and Detonation Physics Research, Institute of Fluid Physics, Chinese Academy of Engineering Physics, Mianyang 621900, China

\begin{abstract}
The high pressure phase transitions of Mo and Ta were investigated experimentally. More melting temperatures were obtained by shock wave experiments. The measured melting temperature at lowest pressure is still much higher than that of DAC experiments. By measurements of sound velocities of Ta in reverse-impact shock wave experiments, a discontinuity of longitudinal sound velocity against shock pressure at $\sim 60 \mathrm{GPa}$ was observed. It may be concluded that a solid-solid phase transition exists.
\end{abstract}

\section{Introduction}

Molybdenum (Mo) and Tantalum (Ta) are two of the elements forming the basis of the ultrahigh pressure scale. The equation of state (EOS) of them at high pressure is being used as a calibration standard to the ruby fluorescence in diamond anvil cell (DAC) experiments [1]. Scientific investigations on the phase transitions for transition metals $\mathrm{Ta}, \mathrm{Mo}$, W have been extensively conducted experimentally and theoretically, because of their enormous discrepancies in melting curves between laser-heated DAC [2-5] and shock wave (SW) [6,7] methods. As for Mo (as well as Ta and W), several thousand degrees of discrepancies exist in extrapolating from DAC pressures of around $100 \mathrm{GPa}$ to SW pressure of $390 \mathrm{GPa}$. For Ta, it also belongs to bec structure at ambient conditions. So far, no bulk phase transitions before melting have been observed by in-situ x-ray diffraction in the DAC experiments up to $174 \mathrm{GPa}[4,5,8]$. The $\mathrm{SW}$ experiments have also confirmed that Ta remains in the bcc phase until shock melting at $300 \mathrm{GPa}$ [9-11]. However, by using transmission electron microscopy, Hsiung and Lassila [12] observed a metastable hexagonal phase in the shear bands of shock-recovered polycrystalline Ta and suggested that a martensitic transformation into the rumpled $\omega$ (simple hexagonal) phase occurred in Ta at $45 \mathrm{GPa}$.

\section{Methods}

To obtain more melting temperature $\left(T_{m}\right)$ data by SW experiments, two series of experiments have been performed. One is using the technique developed by Tan et al. [13] to measure an additional shock-induced release $T_{m}$ data of Mo. The other is using porous Mo in SW experiments, with average initial density $9.557 \mathrm{~g} / \mathrm{cm}^{3}$, as the sample material. The details of the experimental technique can be seen in the previous work [14]. The direct reverse-impact configuration together with the velocity interferometer system for any reflector (VISAR) was employed to measure high-pressure sound velocities in shock-loaded Ta. The detailed experimental technique can be seen in previous work [15].

\footnotetext{
*Project supported by the National Natural Science Foundation of China (Grant No. 10776029).
}

a e-mail: Cai_Lingcang@yahoo.com.cn

This is an Open Access article distributed under the terms of the Creative Commons Attribution-Noncommercial License 3.0, which permits unrestricted use, distribution, and reproduction in any noncommercial medium, provided the original work is properly cited. 


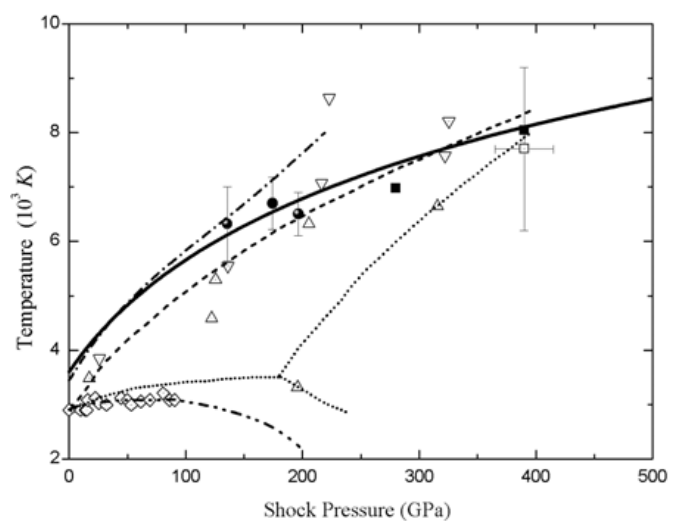

Fig. 1. Melting of Mo. Solid square: our calculated shock-induced equilibrium $T_{m}$ for solid Mo (at about $390 \mathrm{GPa}$ ) and porous Mo (at about $280 \mathrm{GPa}$ ). Open diamond: DAC's $T_{m}$ data [4]; Solid sphere: shock-induced release $T_{m}$ for solid Mo (this work); Semi-solid sphere: shock-induced release $T_{m}$ for porous Mo at about $197 \mathrm{GPa}$ (sapphire window) and at about $136 \mathrm{GPa}$ ( $\mathrm{LiF}$ window); Solid line: our proposed melting curve; Open up- and downtriangles: solid and liquid Mo from ab initio MD simulations [20]; Dash-dotted line: generalized pseudo-potential calculations [21]; Dashed line: ab initio calculations [22]; Dash-dot-dotted line: semi-empirical model [23]; Short dot lines (except the downwards branch): the two-segment melting curve proposed by [16].

\section{Results and discussion}

\subsection{Melting properties of Mo}

With LiF used as the transparent window, we obtained a shock-released melting temperature (174 GPa, $6699 \pm 482 \mathrm{~K}$ ) of Mo. The Hügoniot $C$ results of the porous Mo show that at about $280 \mathrm{GPa}$, the measured Hügoniot $C$ transformed from longitudinal sound wave $C_{l}$ to bulk sound wave $C_{b}$, and $280 \mathrm{GPa}$ is the shock-induced equilibrium melting pressure. Using the thermodynamic calculations, the obtained $T_{m}$ for porous Mo is $6978 \mathrm{~K}(280 \mathrm{GPa})$. Two $T_{m}$ data of porous Mo $(136 \mathrm{GPa}, 6320 \pm 682 \mathrm{~K})$ and $(197 \mathrm{GPa}, 6503 \pm 397 \mathrm{~K})$ were measured, which are given in Fig. 1 . The new $T_{m}$ data measured in our work all agree with our melting curve from Lindemann law. However, in the pressure range close to $100 \mathrm{GPa}$, there still exists large discrepancy in $T_{m}$ data measured between DAC $[2-4,16,17]$ and SW [6] experiments. Furthermore, the $d T_{m} / d P$ behavior does not significantly change when $P-T_{m}$ locus evolves across the triple point of $b c c-h c p$-liquid, at about $210 \mathrm{GPa}$, conjectured by Errandonea [16] from Hixson's Hügoniot $C$ measurements [7]. The melting curve in this work is against the twosegment melting curve model proposed by Errandonea [16].

\section{Solid-Solid phase transition of Ta}

The determined sound velocities are plotted against pressure in Fig. 2. The estimated longitudinal and bulk sound velocities, deduced from Grüneisen EOS and the assumption of $\rho \gamma=\rho_{0} \gamma_{0}=$ constant are also displayed in Fig. 2. In combination with the sound velocity data reported in the literatures $[6,18,19]$, we can clearly see that there are two breaks in the plot of sound velocity against shock pressure at $\sim 60 \mathrm{GPa}$ and $\sim 295 \mathrm{GPa}$. It has been well accepted that the discontinuity at $\sim 295 \mathrm{GPa}$ is resulted from shock-induced melting [6]. Another discontinuity at $\sim 60 \mathrm{GPa}$, however, is observed for the first time. On the basis of the precision of experimental results, we infer that the discontinuity at $\sim 60 \mathrm{GPa}$ likely implies a structural transformation of Ta as the result of Hsiung's shock recovery experiment [12], although it is still impossible to directly determine the structure of the new phase in SW experiments. 


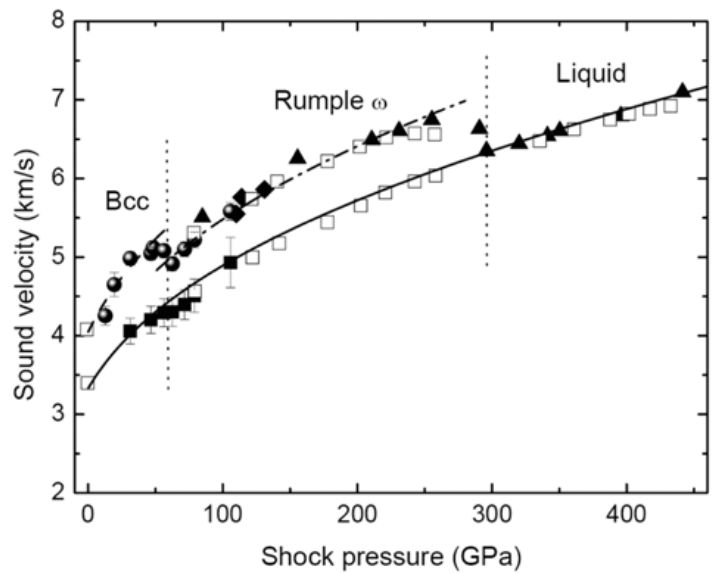

Fig. 2. Sound velocities of Ta. Solid line: the calculated $C_{b}$. Dash line: the calculated $C_{l}$ of bcc Ta. Dash dot line: the calculated $C_{l}$ of rumpled $\omega$ phase. Solid square: $C_{b}$ in this work. Semi-solid sphere: $C_{l}$ in this work. Solid triangles: Ref. [6]. Solid diamond: Ref. [19]. Open square: Ref. [18].

\section{Conclusions}

We investigated the high pressure phase transition properties in Mo and Ta experimentally. The SW experiments were performed to replenish more melting temperature data of Mo. We measured the Hügoniot sound velocity for porous Mo and shock-induced release melting temperature for both solid and porous Mo. The obtained $T_{m}$ data at the lowest pressure are still much higher than that of the DACs and the overall trend of these $T_{m}$ data is against the two-segment melting curve model. By accurate measurements of sound velocities of Ta in SW experiments, a discontinuity of longitudinal sound velocity against shock pressure at $\sim 60 \mathrm{GPa}$ was observed, from which it may be concluded that a solid-solid phase transition exists.

\section{References}

1. H.K. Mao, P.M. Bell, J.W. Shaner, D.J. Steinberg, J. Appl. Phys. 49, 3276 (1978)

2. D. Errandonea, J. Phys.: Condens. Matter 16, 8801 (2004)

3. D. Errandonea, J. Phys. Chem. Solids 67, 2018 (2006)

4. D. Errandonea, B. Schwager, R. Ditz, C. Gessmann, R. Boehler, M. Ross, Phys. Rev. B 63, 132104 (2001)

5. D. Errandonea, M. Somayazulu, D.H. Usermann, H.K. Mao, J. Phys.: Condens. Matter 15, 7635 (2003)

6. J.M. Brown, J.W. Shaner, Shock Waves in Condensed Matter (Elsevier, New York, 1984).

7. R.S. Hixson, D.A. Boness, J.W. Shaner, J.A. Moriarty, Phys. Rev. Lett. 62, 637 (1989)

8. H. Cynn, C.-S. Yoo, Phys. Rev. B 59, 8526 (1999)

9. N.C. Holmes, J.A. Moriarty, G.R. Gathers, W.J. Nellis, J. Appl. Phys. 66, 2962 (1989)

10. A.C. Mitchell, W.J. Nellis, J. Appl. Phys. 52, 3363 (1981)

11. W.J. Nellis, A.C. Mitchell, D.A. Young, J. Appl. Phys. 93, 304 (2003)

12. L.M. Hsiung, D.H. Lassila, Acta mater. 48, 4851 (2000)

13. H. Tan, C. Dai, L. Zhang, C. Xu, Appl. Phys. Lett. 87, 221905 (2005)

14. X.-L. Zhang, L.-C. Cai, J. Chen, J.-A. Xu, F.-Q. Jing, Chin. Phys. Lett. 25, 2969 (2008)

15. J. Hu, X. Zhou, C. Dai, H. Tan, J. Li, J. Appl. Phys. 104, 083520 (2008)

16. D. Errandonea, Physica B 357, 356 (2005)

17. D. Santamaría-Pérez, M. Ross, D. Errandonea, G.D. Mukherjee, M. Mezouar, R. Boehler, J. Chem. Phys. 130, 124509 (2009) 
18. D. Orlikowski, P. Soderlind, J.A. Moriarty, Phys. Rev. B 74, 054109 (2006)

19. Y. Yu, H. Tan, J. Hu, C. Dai, D. Chen, Explosion and Shock Waves (in Chinese) 26, 486 (2006)

20. A.B. Belonoshko, S.I. Simak, A.E. Kochetov, B. Johansson, L. Burakovsky, D.L. Preston, Phys. Rev. Lett. 92, 195701 (2004)

21. J.A. Moriarty, Phys. Rev. B 94, 12431 (1994)

22. C. Cazorla, M.J. Gillan, S.T.e. al., J. Chem. Phys. 126, 194502 (2007)

23. M. Ross, R. Boehler, S. Japel, J. Phys. Chem. Solids 67, 2178 (2006) 